\title{
Ensayo \\ (Essay) \\ RESERVAS DE LA BIOSFERA: PROBLEMAS Y OPORTUNIDADES EN MÉXICO
}

\author{
Gonzalo HaLFFTER \\ Instituto de Ecología, A. C. Carretera antigua a Coatepec No. 351, El Haya, Xalapa, 91070, Veracruz, \\ México. E-mail: gonzalo.halffter@inecol.edu.mx
}

Halffter, G. 2011. Reservas de la Biosfera: Problemas y Oportunidades en México. Acta Zool. Mex. (n. s.), 27(1): 177-189.

RESUMEN. En los últimos dieciséis años el Gobierno Federal Mexicano ha dado una importancia creciente a las áreas naturales protegidas y en especial a las reservas de la biosfera. En este ensayo examinamos cómo este apoyo ha influido en que las reservas puedan cumplir las distintas funciones que les asigna la Estrategia Sevilla, promovida por UNESCO y a la que se han adherido los países miembros. En relación a las capacidades demostradas para conservar la biodiversidad y disminuir el ritmo de cambio del uso del suelo, las primeras informaciones publicadas son contradictorias, aunque en general no muy optimistas. En relación al conocimiento de la biodiversidad y la ecología de los ecosistemas, algunas de las reservas (especialmente aquellas que tienen laboratorio o instituciones de investigación asociados) han sido centros muy importantes de investigación, generándose un cúmulo realmente importante de conocimientos. La integración y participación de las comunidades locales está en vías de mejorar, aunque hay varios aspectos no suficientemente desarrollados y en más de un caso persiste una aceptación limitada por parte de las poblaciones locales. Hay que anotar que en los últimos años se ha logrado un avance muy importante en el pago por servicios ambientales. No se ha cumplido (principalmente por falta de integración con otros programas gubernamentales de desarrollo) la recomendación de que las reservas de la biosfera sirvan de laboratorios para la búsqueda de nuevas alternativas que favorezcan el desarrollo sustentable a nivel regional.

Convencido de que no se logrará una conservación efectiva de la biodiversidad si no se presta atención a lo que ocurre fuera de las áreas naturales protegidas, la última parte del ensayo está dedicada a las propuestas de conservación campesinas, ejidales, comunitarias o privadas.

Palabras clave: Reservas de la biosfera, México, poblaciones locales, estímulos para la conservación.

Halffter, G. 2011. Biosphere Reserves: Problems and oportunities in México. Acta Zool. Mex. (n. s.), 27(1): 177-189.

ABSTRACT. Over the last sixteen years the Federal Government of Mexico has given growing importance to protected natural areas, and particularly the biosphere reserves. In this essay we examine the

Recibido: 26/08/2010; aceptado: 05/11/2010. 
effect of this support on the biospheres' fulfilling the different functions outlined by the Seville Strategy, promoted by UNESCO and adopted by member countries. The first reports published on the biodiversity and ecosystem ecology protection in these areas are contradictory and not, in general, very optimistic. Some reserves - especially those that have a laboratory or are associated with a research institute - are very important research centres, and have made a truly important contribution to our knowledge. The incorporation and participation of local communities is improving although there are several aspects that are not yet well developed and in more than one instance there is limited acceptance by the local population. In recent years, there has been notable headway with payment for environmental services. The recommendation that biosphere reserves serve as laboratories for in the search for new alternatives that favour sustainable development at the regional level has not been met; mainly because of the lack of cooperation with other government development programs. It is evident that the biodiversity cannot be effectively protected if no attention is paid to what is happening outside the protected natural areas, thus the last part of the essay deals with rural, ejido, community and private conservation proposals.

Key words: biosphere reserve, Mexico, local population, incentives for conservation.

\section{INTRODUCCIÓN}

\section{Antecedentes históricos}

A diferencia de otros tipos de áreas de conservación in situ, las reservas de la biosfera corresponden a un concepto creado, discutido y modificado por científicos, con el propósito de crear una alternativa distinta, pero no excluyente, a los parques nacionales y similares. Una alternativa que contemple los requerimientos de la conservación de la biodiversidad, pero también las realidades económicas y sociales de nuestros días. Una alternativa que conjugue conservación del patrimonio natural con desarrollo sustentable. Esta alternativa se crea y desarrolla en el Programa Hombre y Biosfera (MAB) de UNESCO.

En su primera versión (1974) se planteaba crear una red internacional de áreas protegidas que conservasen el germoplasma bajo un enfoque sistémico (es decir, considerando a los ecosistemas como unidades) y que privilegiase la investigación científica. En 1976, se establece la primera lista de reservas aceptadas por MAB-UNESCO. En aquellos años de guerra fría, la Unión Soviética y los Estados Unidos compitieron estableciendo reservas de la biosfera, para lo cual simplemente cambiaron de denominativo a muchos de sus principales parques nacionales. Se crea así una confusión entre los propósitos de los parques y los de las reservas. Confusión sólo aparente, pues lo que legal y realmente existía a nivel de país eran los parques nacionales.

En México, la creación de las primeras reservas siguió otro camino. La situación y administración de los parques nacionales era muy defectuosa, cuando no nula (véase Carabias et al. 2008; Simonian 1999). Se trataba de buscar algo distinto. En estas circunstancias una serie de centros de investigación (en primer término histórico el Instituto de Ecología, A.C.) se propusieron crear reservas de la biosfera en estrecha colaboración con los correspondientes Gobiernos Estatales, y contando con el 
apoyo económico de proyectos de CONACYT. Así se establecieron las reservas de Mapimí y La Michilía (Durango), El Cielo (Tamaulipas), Manantlán (Jalisco), Sian Ka'an (Quintana Roo), Montes Azules (Chiapas), y como una acción específica de la UNAM, el área protegida que después ha sido parte de la reserva de Los Tuxtlas, Veracruz (para información sobre reservas de la biosfera en México, véase GómezPompa y Dirzo 1995; Simonian 1999).

En el proceso para establecer estas primeras reservas se buscó la participación de las poblaciones locales y se incluyeron dentro de los programas de trabajo además de proyectos de investigación básica, otros que pudieran beneficiar directamente a las poblaciones locales, ejemplos serían los estudios para el manejo del agua y la ganadería en Mapimí, o para un uso forestal más sustentable en Manantlán (Halffter 1984a y b, 2002).

En 1983 se celebró en Minsk (Bielorrusia) la Primera Conferencia Internacional sobre reservas de la biosfera. En esta conferencia, en la que varios científicos mexicanos tuvimos una importante contribución, quedó claro que había dos ideas muy diferentes sobre lo que debía ser una reserva de la biosfera: por un lado la reserva tipo parque; por otro el modelo que se venía experimentando en México y que sería conocida como "modalidad mexicana", con énfasis en la participación local y regional.

En la Segunda Conferencia Internacional sobre reservas de la biosfera, celebrada en Sevilla en 1995, la situación había cambiado por completo: la participación y las necesidades de las poblaciones locales, la investigación para el desarrollo sustentable y la restauración de la biodiversidad en áreas degradadas, quedaron totalmente incorporadas en la muy importante declaración que emanó de la Conferencia: la Estrategia Sevilla.

La Estrategia Sevilla se centra en un desarrollo sustentable que incluya la salvaguarda del ambiente y su riqueza biótica y una mayor equidad social, incluyendo el respeto a los usos tradicionales de las comunidades locales. Busca promover simultáneamente conservación y desarrollo sustentable. Más que "islas de conservación", las reservas deberán ser laboratorios para reconciliar las necesidades del hombre con la conservación de la naturaleza. Se sufragan dos propósitos muy importantes: poner en valor los conocimientos tradicionales, y servir de referencia regional para sobrepasar las dificultades derivadas de distintos programas e intereses regionales.

Para que los distintos propósitos antes enunciados puedan ser puestos en práctica, dos cosas son indispensables. La primera, establecer una zonación clara dentro de cada reserva, que permita distinguir aquellas áreas destinadas exclusivamente a la conservación y la investigación científica no manipulativa (Zonas Núcleo), de aquellas destinadas a la restauración ecológica y a la búsqueda de alternativas sustentables de uso de los recursos bióticos. El segundo punto esencial es que las reservas no podrían ser espacios vacios de población humana. Sin poblaciones locales no era posible la búsqueda de las nuevas alternativas con sentido biológico, pero también social. 
Muy ilustrativo de la Estrategia Sevilla es el señalamiento de que la gestión de cada reserva debe verse como "un pacto" entre las comunidades locales y la sociedad en su conjunto, representada por sus respectivos gobiernos.

Con la Estrategia Sevilla por fin las reservas de la biosfera tienen un documento internacional aceptado por todos los países miembros de UNESCO. Se plantea pasar de una conservación pasiva en la que la prioridad es no intervenir en los procesos naturales, a una conservación activa que incluye la restauración y el uso sustentable. Las muchas experiencias que para entonces se habían implementado en varias reservas, junto con la divulgación de la Estrategia han tenido una influencia que rebasa la red de reservas, cambian en muchos casos la gestión de los parques e influyendo en la creación de áreas protegidas comunitarias, privadas, urbanas o periurbanas (Halffter et al. 2007).

En febrero 2008 se celebró en Madrid la Tercera Conferencia Internacional de reservas de la biosfera. Su propósito fue determinar qué había pasado con la aplicación de la Estrategia Sevilla (UNESCO-MAB 2008). La recomendación principal fue insistir en el papel de las reservas para promover el desarrollo sustentable.

Actualmente el Programa MAB-UNESCO, coordinador internacional de las reservas de la biosfera, se apoya en tres ejes de acción: 1) Reducir al mínimo la pérdida de biodiversidad a través de la investigación y la creación de capacidades en materia de gestión; 2) Promover la sustentabilidad del medio ambiente; 3) Fortalecer los vínculos entre la diversidad cultural y la diversidad biológica.

\section{Las reservas y el problema global de conservación de la biodiversidad}

Hay una evidencia creciente de que a pesar del aumento considerable de reservas de la biosfera y otras áreas protegidas, una parte muy importante de la biodiversidad queda y seguirá quedando fuera de los límites de estas áreas. Esto es especialmente importante en paisajes tropicales en los que la diversidad beta (o sea el recambio espacial de especies) es un componente muy significativo de la diversidad total. Por lo anterior, se ha insistido (véase Halffter 2005, Allen et al. 2003, Chazdon et al. 2009 y bibliografía ahí citada) en que debe prestarse atención a lo que ocurre fuera de las áreas protegidas, en especial a la conservación ligada al uso tradicional de los recursos bióticos. Una expresión legal de este interés por la conservación asociada al uso no intensivo, es la formalización por parte del Gobierno Mexicano de las áreas de protección voluntario-comunitarias a las que me refiero al final del ensayo.

Estoy totalmente de acuerdo con Sarukhan et al. (2009: 79) cuando señalan: "La conservación efectiva de la diversidad biológica mexicana solo podrá darse, con contadas excepciones, en el contexto de lograr un uso sustentable del capital natural por parte de las poblaciones que han habitado los territorios bajo protección durante generaciones." 
Sobre el mismo punto, Guevara y Halffter (2007) dicen: “... la conservación de la biodiversidad como un componente indispensable del desarrollo sustentable, rebasa las fronteras de las reservas de la biosfera. Las reservas están inmersas en un paisaje mucho más amplio. Un paisaje que para ser sustentable deberá armonizar los usos de la diversidad biológica, del suelo y del agua. El conjunto resultante debe contribuir tanto al bienestar de la sociedad y al progreso económico, como a la preservación de los procesos naturales y ecológicos de los que depende la vida del planeta."

\section{LAS RESERVAS DE LA BIOSFERA EN MÉXICO}

En México, desde 1994 la posición del Gobierno Federal en relación a las áreas naturales protegidas, incluyendo las reservas de la biosfera, ha dado un giro de $180^{\circ}$ que venía preparándose. La actuación en puestos muy importantes de Julia Carabias, Javier de la Maza y Ernesto Enkerlin, ha sido por demás notable. Se ha creado (2000) una entidad administrativa de alto nivel: la Comisión Nacional de Áreas Naturales Protegidas (CONANP) (para antecedentes y organización de la CONANP véase Bezaury-Creel y Gutiérrez Carbonell 2009). Así mismo, de una manera gradual y después de un análisis y revisión detallados, se han ido incorporando las áreas ya decretadas a un Sistema Nacional de Áreas Naturales Protegidas que sirve como base de referencia.

Muchas de las áreas, entre ellas reservas de la biosfera, han sido dotadas de personal administrativo y presupuesto. Por otra parte, México es uno de los pocos países que incluyen en su legislación la figura de reserva de la biosfera (Ley General del Equilibrio Ecológico y Protección del Ambiente, Arts. 46 y 47).

El Gobierno Federal ha tomado a su cargo la protección de las reservas de la biosfera ya existentes y la creación de nuevas. De las 172 áreas naturales protegidas que existen bajo distintas categorías, 39 son reservas de la biosfera con una superficie total de casi 12 mill. de has. Lo anterior sitúa a México entre los países con más reservas de la biosfera (CONANP 2006, 2007, 2008).

La superficie decretada como área natural protegida (incluyendo las reservas de la biosfera) indica la prioridad que tiene la conservación en la agenda gubernamental, pero no necesariamente señala el grado de efectividad con el que están siendo protegidos los ecosistemas y la biodiversidad del país. Evaluar las áreas protegidas involucra al menos dos preguntas: ¿Es la cobertura actual adecuada y suficientemente representativa para proteger los ecosistemas a nivel nacional? ¿Cuál ha sido el impacto del establecimiento de las áreas naturales protegidas en la conservación de los ecosistemas y la biodiversidad?

Según los datos reunidos en el excelente estudio de Urquiza (2009), la representación de los ecosistemas prioritarios en el sistema nacional de áreas naturales protegidas deja mucho que desear, ya que sólo un $12.9 \%$ se encuentra formalmente protegido. La evaluación de la efectividad en la conservación cuenta con datos apenas 
iniciales. Según un estudio realizado por la CONANP en 2006 (véase Urquiza 2009) de 14 áreas estudiadas, 11 fueron valoradas negativamente como resultado de altas tasas de transformación que se reflejan en pérdidas de cobertura vegetal. Sin embargo, otros estudios son mucho más optimistas (véase Sánchez Cordero y Figueroa 2007; González Montagut 2009 y Sánchez Cordero et al. 2009. El último trabajo señalado incluye un análisis cuantitativo para evaluar la capacidad que han mostrado las áreas protegidas para conservar la vegetación primaria y disminuir el incremento de la superficie transformada). Es de señalar que según los trabajos antes mencionados, el tipo de área con mayor proporción de resultados positivos ha sido las reservas de la biosfera.

\section{¿CÓMO HA AFECTADO AL FORTALECIMIENTO ADMINISTRATIVO LAS DISTINTAS FUNCIONES QUE DEBEN REALIZAR LAS RESERVAS DE LA BIOSFERA?}

\section{Investigación científica}

En lo que se refiere a la investigación científica, Mapimí, Los Tuxtlas, Chamela, Manantlán y en menor escala La Michilía y Montes Azules, cuentan con instalaciones para la estancia y trabajo de investigadores. Los resultados son evidentes. La cantidad de información, publicaciones y libros generados convierte a estas reservas en referentes a nivel nacional e incluso internacional del conocimiento sobre biodiversidad y ecología tropical.

Al fortalecer el aparato administrativo, las autoridades superiores de la Comisión han entendido bien y apoyado el papel que juegan los centros de investigación, en muchos casos incluso se han firmado convenios formales de cooperación. Pero esto no siempre ha ocurrido a nivel de los directores locales. Especialmente cuando se trata de programas de investigación para el desarrollo que incluyen interacciones con poblaciones y organizaciones locales.

Hay varios aspectos de la actividad científica, especialmente de aquella dirigida a problemas locales, que hasta ahora no se han explotado debidamente. Me refiero a utilizar las reservas como laboratorios para la búsqueda de alternativas de desarrollo sustentable que puedan proyectar sus resultados a nivel regional y que sean tomadas en cuenta en los programas de desarrollo estatal y regional. No es sencillo, como en ningún caso lo es la transferencia de nuevos conocimientos a la actividad económica, pero si es factible y podría dar a las reservas una presencia pública importante.

Otra actividad científica que hasta donde yo sé no se ha realizado, es el uso de las reservas y todo el conocimiento y facilidades que ofrecen como observatorios para medir a largo plazo el cambio global. Se trata de algo importante y actual, pero que necesita de fondos y estrategias específicas. 


\section{Interacción con las poblaciones locales}

¿Qué ha pasado con la interacción con las poblaciones? Sin duda, el costo en restricciones por no uso de los recursos recae en las poblaciones locales. En su mayor extensión, las reservas de la biosfera de México, incluyendo sus zonas núcleo en las que no puede haber ninguna actividad económica, siguen siendo de propiedad ejidal, comunal o privada. Sabemos que los territorios en los que habitan pueblos indígenas no solamente contienen una buena parte de la diversidad del país, sino el $26.2 \%$ de las áreas protegidas (Sarukhan et al. 2009).

Según Bezaury-Creel y Gutiérrez Carbonell (2009) en todo el país, en el año 2000 había 1562 ejidos y comunidades agrarias (5.2\% de los existentes en México) con porciones de su superficie comprendidas en las áreas naturales protegidas creadas por decreto federal. Según información actualizada a fines de 2004, 1879 núcleos agrarios se ubican total o parcialmente en áreas naturales protegidas federales, 1385 en áreas naturales protegidas estatales y 95 en las de designación municipal. Es por demás evidente que como lo han señalado algunos autores (véase en especial Boege 2009; Toledo y Barrera-Bassols 2008) la conservación de la biodiversidad en México (y en muchos otros países) no puede lograrse sin una participación activa de las poblaciones locales y pueblos indígenas.

¿En qué forma las poblaciones locales y los pueblos indígenas participan en la creación y gestión de las áreas protegidas y qué beneficios reciben a cambio de las restricciones que se les imponen?

La CONANP ha señalado que en 2006, 90\% de las áreas prioritarias contaban con acciones de fortalecimiento de la participacion social e institucional. Además, la Procuraduría Federal de Protección al Ambiente ha trabajado en la integración de comités de vigilancia social participativa, que en 2006 sumaban 104 (SEMARNAT 2006).

Según las encuestas que reúne Urquiza (2009) a pesar de estos esfuerzos, persiste una limitada aceptación de las áreas protegidas por parte de las comunidades locales. Lo anterior lo atribuye, entre otras cosas, a la imposición unilateral del decreto y del plan de manejo, práctica que parece tiende a desaparecer, así como a la frecuente sensación de exclusión de las comunidades locales en la toma de decisiones. A pesar del desarrollo de mecanismos de participacion social, éstos no siempre se cumplen y la exclusión (o la sensación de exclusión) fomenta la percepción de pérdida de tutoría sobre su propiedad por parte de las poblaciones locales.

Cuando se imponen restricciones al uso de recursos sin promover otras alternativas productivas, se reduce la aceptación social pues se afecta de modo inmediato el estilo y calidad de vida de las poblaciones locales. En algunos casos, lo anterior al acentuar la sensación de despojo ha llevado a incrementar drásticamente el uso del recurso, con el fin de garantizar los beneficios inmediatos.

Existen varios estudios sobre la precepción de las reservas de la biosfera y sus actividades por las poblaciones locales: reserva de Mapimí (Kaus 1993); reserva de Los 
Tuxtlas (Durand 2002); reserva de Chamela-Cuixmala (Castillo et al. 2007) y reserva de Ría Lagartos (Fraga 2006).

En cuanto a los beneficios, en algunos casos los ha habido, especialmente en aquellas reservas de la biosfera costeras que tienen un fuerte atractivo turístico. Incluso fuera de las costas, en otros casos también ha habido beneficios por el turismo. Es el caso del ecoturismo organizado por comunidades indígenas en fragmentos de selva en la periferia de la reserva de Montes Azules, Chiapas (Navarrete y Halffter 2008) y en algunas otras reservas de la biosfera.

Un caso interesante es la organización de los campesinos para el cultivo (en lugar de extracción clandestina) de cactus en la reserva de Tehuacán - Cuicatlán. En el pequeño poblado de Zapotitlán Salinas, situado al lado del centro de acogida del área de Tehuacán, el cultivo de cactus y los servicios de guías para recorrer la reserva son actividades que se dejan sentir económicamente y que crean un buen ambiente hacia la propia reserva.

\section{PAGO POR SERVICIOS AMBIENTALES}

La idea que subyace en el pago por servicios ambientales es que quienes se benefician de ellos deberían pagar a quienes conservan y protegen los ecosistemas que los proveen. Muy posiblemente es la forma más general de compensar a las poblaciones locales de las restricciones que estar en un área protegida (y más si es un área núcleo) imponen. El pago debe llegar a quienes restringiendo o evitando el cambio de uso del suelo, protegen y conservan la biodiversidad (para una revisión de los distintos instrumentos territoriales y económicos que favorecen la conservación y el uso sustentable de la biodiversidad, véase Alvarez-Icaza et al. 2008).

El programa de pago por servicios ambientales del Gobierno Federal Mexicano comenzó en 2003. Los pagos por servicios hidrológicos fueron los primeros en ser establecidos. Se refieren a la retribución a los propietarios de predios forestales por desarrollar acciones de protección y manejo de ecosistemas con el fin de mantener o mejorar la provisión de agua (CONAFOR 2008). Según Urquiza (2009) con este sistema de pagos se cubría a la fecha una superficie de 1370516 ha. (0.70\% del territorio nacional).

El pago por captura de carbono se inició en 2004 e incluye actividades de forestación y reforestación. El programa no exige que las especies utilizadas sean nativas, ya que pueden utilizarse especies exóticas. A la fecha se ha forestado a reforestado 319 673 ha $(0.16 \%$ del territorio nacional).

El pago de servicios ambientales por biodiversidad está encaminado a acciones de protección y manejo de la biodiversidad. Se da prioridad a aquellas actividades que se realizan en áreas naturales protegidas, sitios Ramsar, áreas de importancia para la conservación de las aves o con especies en alguna categoría de riesgo. Según Urquiza 
(2009) a nivel nacional un total de 694620 ha se encuentra inscrita dentro de este programa.

El pago por sistemas agroforestales con cultivos bajo sombra, contempla el apoyo a actividades que siendo productivas ayudan a mantener la calidad del ambiente (por ejemplo, cultivo del café bajo sombra con árboles nativos). Bajo este esquema se encuentran incluidas 462207 ha (0.24\% del territorio nacional) (Urquiza 2009).

En la actualidad (2010) los distintos programas de pagos por servicios ambientales antes mencionados, se han agrupado en el programa PROARBOL manejado por la Comisión Nacional Forestal (CONAFOR), con una estrecha asociación de la CONANP.

Resulta muy ilustrativo establecer la relación entre pagos por servicios ambientales y áreas naturales protegidas. Según información de Francisco Javier Medina (in litt. 30/IV/2010), la CONANP ha logrado atraer recursos considerables del programa PROARBOL, beneficiando directamente a los habitantes de áreas naturales protegidas. De 2003 a 2008 se han beneficiado 75 áreas (397 839 ha) con pagos relativos al menos a uno de los servicios a que se refiere el programa. Según la misma fuente, entre 2003 y 2009 del conjunto de pagos por servicios ambientales, el 30\% de ha ejercido en áreas naturales protegidas, porcentaje que sube a $40-50 \%$ si consideramos sólo el pago por servicios hidrológicos.

Aunque el programa por servicios ambientales no está dirigido exclusivamente a áreas naturales protegidas (se toman en cuenta otros elementos como combate a la pobreza), constituye una acción muy importante para reforzar la conservación en términos de desarrollo sustentable dentro de las áreas. A pesar de que el programa ha ido creciendo en importancia económica, es indudable que el monto de dinero que maneja es insuficiente para provocar un cambio importante hacia usos más sustentables de los recursos bióticos. También sería deseable aumentar el porcentaje que se dedica a áreas naturales protegidas, no sólo por razones evidentes de conservación, sino como parte de una política integral para lograr una mayor aceptación de las áreas protegidas entre las poblaciones locales.

En el momento actual (pero hay que tomar en cuenta el poco tiempo que tiene el programa funcionando), no se sabe con certeza si los pagos otorgados reducen o no la presión para cambio de uso del suelo. En general (véanse ejemplos en Urquiza 2009) los pagos son inferiores a lo que se produciría en uso extractivo o convencional.

Otro aspecto a desarrollar. Sabemos que México es el lugar de origen de numerosas plantas domesticadas y de sus variedades próximas. Pero no se ha planteado utilizar las zonas de influencia de aquellas reservas que se prestan para ello, para fomentar el cultivo de las variedades nativas locales, siguiendo las metodologías tradicionales. Una acción de este tipo tendría dos ventajas: por un lado se incrementaría las posibilidades de sobrevivencia in situ de las variedades nativas, pero, además con un mínimo financiamiento se podría establecer una fuente adicional de recursos para 
las poblaciones locales, con el consiguiente involucramiento en las actividades de la reserva.

\section{ÁREAS DE PROTECCIÓN VOLUNTARIA-COMUNITARIA}

En los últimos años se han reconocido formalmente o propuesto, otras alternativas de área natural protegida que coinciden en sus objetivos con las reservas de la biosfera. Se trata de las reservas comunitarias, campesinas, ejidales o privadas que quedan agrupadas bajo el término de áreas de conservación voluntaria-comunitaria. Es el reconocimiento de una práctica que en México y muchos otros países viene realizándose desde tiempos ancestrales como parte de una visión de uso rústico-conservación (Allen et al. 2003; Halffter 2005; Boege 2008; Toledo y Barrera-Bassols 2008; Bezaury-Creel y Gutierrez Carbonell 2009).

Al contrario de las propuestas basadas en una decisión gubernamental, este tipo de área protegida tiene sus bases en los valores culturales y modos de vida tradicional, un tema ampliamente tratado en la bibliografía antes citada. Se caracterizan por ser espacios que voluntariamente excluyen los propietarios de las actividades productivas de alto impacto como la ganadería y la agricultura.

En México se han realizado varios recuentos importantes de las iniciativas de conservación comunitaria (Anta y Pérez 2004; Anta y Pérez 2006). A la fecha (CONANP 2009) están registradas 191 áreas de este tipo, en 15 estados, con una superficie total de 242852 has. Muy interesante es que entre los propietarios participan nueve grupos étnicos, lo que involucra unas 70,000 personas.

Sintetizando la clasificación de este tipo de áreas propuesta por Urquiza (2009), tenemos: 1) Áreas de conservación creadas para mantener o recobrar el control de los recursos. Se han impulsado con el fin de proteger recursos o espacios en conflicto de propiedad o de degradación y explotación por terceros. El ejemplo más conocido es la Reserva Campesina de Los Chimalapas en Oaxaca.

2) Áreas de conservación con fines económicos. En estos casos se destinan ciertos espacios a la producción de bienes y servicios no agresivos con el medio ambiente, excluyéndolos de los usos productivos convencionales, lo que promueve su conservación. En estas condiciones se encuentran las Unidades de Manejo para la Conservación de la Vida Silvestre (UMAS), las áreas que reciben pagos por servicios ambientales, las áreas con cultivo de café orgánico y bajo sombra, las destinadas al ecoturismo, etc.

3) Áreas de conservación como subproducto o subcomponente de otro proceso. Hay varios ejemplos de áreas comunitarias que se dedican a la conservación como resultado de un requerimiento externo o a partir de otros proyectos y programas. Sería el caso de áreas establecidas a partir de ordenamientos territoriales comunitarios, o como resultado de los requerimientos para obtener la certificación forestal, o de 
programas como el Plan Piloto Forestal y COPLAMAR en la Península de Yucatán. Como ejemplo, a raíz de los procesos de planeación comunitaria del territorio realizados en Oaxaca, se han destinado 54,663 has a zonas de conservación por acuerdo comunitario.

4) Áreas de protección de recursos estratégicos. Se trata de áreas de conservación, reconocidas por la comunidad, que son decretadas o asumidas como tales por acuerdo en asambleas comunitarias o ejidales. Tienen como propósito proteger recursos como manantiales, sitios de recarga de agua, refugios de fauna y sitios de extracción de recursos no maderables o medicinales.

Incrementar este tipo de área voluntaria, perfeccionar los mecanismos legales de apoyo a los poseedores, y buscar formas de financiamiento por parte de ONG's y otras organizaciones similares, tendrá efectos muy importantes en la conservación de la biodiversidad, especialmente si se combina su desarrollo con el de reservas archipiélago. Estas reservas están pensadas para que sean paraguas regionales que permitan incrementar la conectividad biológica entre distintas áreas, así como generar apoyos de cualquier tipo a las áreas ya existentes de cualquier clase, áreas que actualmente no se relacionan entre sí (Halffter 2005).

AGRADECIMIENTOS. Agradezco profundamente la información que me ha sido proporcionada por el Biol. Francisco Javier Medina y la M.C: Pía Gallina, ambos de la CONANP. También la ayuda en la revisión bibliográfica de la Dra. Lucrecia Arellano, Instituto de Ecología, A.C.

\section{BIBLIOGRAFÍA}

Allen, M.F., A. Gómez-Pompa, E. Rodríguez-Luna \& J.J. Jiménez-Osorio. 2003. La conservación de la biodiversidad en áreas no protegidas: un enfoque de redes. En: Hacia una evaluación de las áreas naturales protegidas tropicales. Centro de Investigaciones Tropicales, Universidad Veracruzana, Xalapa, Veracruz, México.

Álvarez Icaza, P. et al. 2008. Instrumentos territoriales y económicos que favorecen la conservación y el uso sustentable de la biodiversidad. En CONABIO. Capital natural de México, Vol. III: 229-258. CONABIO, México, D.F.

Anta, S. \& P. Pérez. 2004. Atlas de experiencias comunitarias en el manejo sostenible de los recursos naturales en Oaxaca. 58 pp. SEMARNAT, México, D.F.

Anta, S. \& P. Pérez. 2006. Atlas de experiencias comunitarias con manejo sostenible en el estado de Quintana Roo. 61 pp. SEMARNAT, México, D.F.

Bezaury-Creel, J. \& D. Gutiérrez Carbonell. 2009. Áreas naturales protegidas y desarrollo social en México. En CONABIO. Capital natural de México, Vol. II: 382-431. Comisión Nacional para el Conocimiento y Uso de la Biodiversidad, México, D.F.

Boege, E. 2008. El patrimonio biocultural de los pueblos indígenas de México: Hacia una conservación in situ de la biodiversidad y agrodiversidad en los territorios indigenas. $342 \mathrm{pp}$. Instituto Nacional de Antropología e Historia y Comisión Nacional para el Desarrollo de los Pueblos Indígenas, México, D.F.

Boege, E. 2009. El reto de la conservación de la biodiversidad en los territorios de los pueblos indígenas. En CONABIO. Capital natural de México, Vol. II: 603-649. Comisión Nacional para el Conocimiento y Uso de la Biodiversidad, México, D.F. 
Carabias, J., J. de la Maza \& E. Provencio. 2008. Evolución de enfoques y tendencias en torno a la conservación y el uso de la biodiversidad. En CONABIO. Capital natural de México, Vol. III: 2942. CONABIO, México, D.F.

Castillo, A., M.A. Magaña. A. Pujadas, L. Martínez \& C. Godínez. 2005. Understanding rural people interaction with ecosystems: A case study in a tropical dry forest of Mexico. Ecosystems, 8: 630643.

Castillo, A., A. Pujadas \& N. Schroeder. 2007. La reserva de la biosfera Chamela-Cuixmala, México: perspectivas de los pobladores rurales sobre el bosque tropical seco y la conservación de ecosistemas. In: G. Halffter, S. Guevara y A. Melic (Eds.). Hacia una cultura de conservación de la diversidad biológica. Pp. 245-254. M3M: Monografías Tercer Milenio, Vol. 6, Sociedad Entomológica Aragonesa, Zaragoza.

Chazdon, R.L., C.A. Harvey, O. Komar, D.M. Griffith, B.G. Ferguson, M. Martínez-Ramos, H. Morales, R. Nigh, L. Soto-Pinto, M. van Brengel \& S.M. Philpott. 2009. Beyond reserves: A research agenda for conserving biodiversity in human-modified tropical landscapes. Biotropica, 41 : 142-153.

CONAFOR. 2008. < www.conafor.gob.mx >

CONANP. 2006. Las áreas protegidas de México. Liderazgo Internacional. Comisión Nacional de Áreas Naturales Protegidas. México, D.F. Pp. 48.

CONANP. 2007. Áreas protegidas de México, Informe Nacional 1997-2007. II Congreso Latinoamericano de Parques Nacionales y Otras Áreas Protegidas, Bariloche, Argentina. Comisión Nacional de Áreas Naturales Protegidas. México, D.F. Pp. 92.

CONANP. 2008. Cooperación Internacional de México y sus Beneficios para la Conservación. Comisión Nacional de Áreas Naturales Protegidas. México, D.F. Pp. 120.

CONANP. 2009. < www.conanp.gob.mx >

Durand, L. 2002. La difusión y percepción del discurso y las practicas ambientalistas en la Sierra de Santa Martha, Veracruz: Un estudio de caso. Centro Regional de Investigaciones Multidisciplinarias, UNAM, Cuernavaca, México.

Fraga, J. 2006. Local perspectives in conservation politics. The case of the Lagartos Biosphere Reserve, Yucatan, Mexico. Landscape and Urban Planning, 74: 285-295.

Gómez-Pompa, A. \& R. Dirzo (Coords.). 1995. Reservas de la biosfera y otras áreas protegidas de México. Instituto Nacional de Ecología - Comisión Nacional para el Conocimiento y Uso de la Biodiversidad, México, D.F.

Gonzalez Montagut, R. 2009. ¿Funcionan las reservas de la biosfera? La experiencia del Fondo para Áreas Naturales Protegidas. In: CONABIO. Capital natural de México, Vol. II: 411-412. Comisión Nacional para el Conocimiento y Uso de la Biodiversidad. México, D.F.

Guevara, S. \& G. Halffter. 2007. Estrategia para la conservación de la diversidad biológica en áreas protegidas de designación internacional: La síntesis. In: G. Halffter, S. Guevara y A. Melic (Eds.). Hacia una cultura de conservación de la diversidad biológica. Pp. 9-18. M3M: Monografías Tercer Milenio, Vol. 6, Sociedad Entomológica Aragonesa, Zaragoza.

Halffter, G. 1984a. Conservation, development and local participation. In: F. di Castri, F.W. Baker y M. Hadley (Eds.). Ecology in Practice, Vol. 1: 428-436. Tycooly International Publishing Limited, Dublin.

Halffter, G. 1984b. Biosphere reserves: The conservation of nature for man. En UNESCO-UNEP. Conservation, Science and Society, Vol. 2: 450-457. UNESCO, París.

Halffter, G. 2002. Conservación de la biodiversidad en el Siglo XXI. Boletín Sociedad Entomológica Aragonesa, 31: 1-17.

Halffter, G. 2005. Towards a culture of biodiversity conservation. Acta Zoológica Mexicana (n.s.), 21: 133-153. 
Halffter, G. S. Guevara \& A. Melic (Eds.). 2007. Hacia una cultura de conservación de la diversidad biológica. M3M: Monografías Tercer Milenio, Vol. 6, 358 Pp. Sociedad Entomológica Aragonesa, Zaragoza.

Kaus, A. 1993. Environmental perceptions and social relation in the Mapimí Biosphere Reserve. Conservation Biology, 7: 398-406.

Navarrete, D. \& G. Halffter. 2008. Dung beetle (Coleoptera: Scarabaeidae: Scarabaeinae) diversity in continuous forest, forest fragments and cattle pastures in a landscape of Chiapas, Mexico: the effects of anthropogenic changes. Biodiversity Conservation, 17: 2869-2898.

Sánchez-Cordero, V. \& F. Figueroa. 2007. La efectividad de las reservas de la biosfera en México para contener procesos de cambio en el uso del suelo y la vegetación. In: G. Halffter, S. Guevara y A. Melic (Eds.). Hacia una cultura de conservación de la diversidad biológica. Pp. 161-171. M3M: Monografías Tercer Milenio, Vol. 6, Sociedad Entomológica Aragonesa, Zaragoza.

Sánchez-Cordero, V., F. Figueroa, P. Illaldi \& M. Linaje. 2009. Efectividad de las áreas naturales protegidas de México. In: CONABIO. Capital natural de México, Vol. II: 394-397. Comisión Nacional para el Conocimiento y Uso de la Biodiversidad, México, D.F.

Sarukhan, J. et al. 2009. Capital natural de México. Sintesis: Conocimiento actual, evaluación y perspectivas de sustentabilidad. 100 pp. Comisión Nacional para el Conocimiento y Uso de la Biodiversidad, México, D.F.

SEMARNAT. 2006. Documento en línea <www.semarnat.gob.mx/queessemarnat/documents/p_conanp.pdf $>$

Simonian, L. 1999. La defensa de la tierra del jaguar. Una historia de la conservación en México. Comisión Nacional para el Conocimiento y Uso de la Biodiversidad - Instituto Nacional de Ecología - Instituto Mexicano de Recursos Naturales Renovables, México, D.F.

Toledo, V.M. \& N. Barrera-Bassols. 2008. La memoria biocultural: La importancia ecológica de las sabidurías tradicionales. 220 pp. Icaria Editorial, Barcelona.

Urquiza Haas, E.G. 2009. Análisis de capacidades nacionales para la conservación in situ. In: CONABIO - PNUD. México: Capacidades para la conservación y uso sustentable de la biodiversidad. Pp. 51-94. Comisión Nacional para el Conocimiento y Uso de la Biodiversidad y Programa de las Naciones Unidas para el Desarrollo, México, D.F. 